\title{
Quadriceps-splitting midline approach in the treatment of distal femur infected nonunion with stiff knee and severely scarred soft tissues
}

\author{
Jeremy Bliss, Dan Barnabas Inja*, Manasseh Nithyananth, Vinoo Mathew Cherian
}

Department of Orthopaedics, Unit 1, Christian Medical College, Vellore, Tamil Nadu, India

Received: 25 January 2020

Revised: 10 February 2020

Accepted: 11 February 2020

\author{
*Correspondence: \\ Dr. Dan Barnabas Inja, \\ E-mail: injadan@yahoo.com
}

Copyright: (C) the author(s), publisher and licensee Medip Academy. This is an open-access article distributed under the terms of the Creative Commons Attribution Non-Commercial License, which permits unrestricted non-commercial use, distribution, and reproduction in any medium, provided the original work is properly cited.

\begin{abstract}
Background: Treatment of infected distal femur non-union with a stiff knee and severely scarred soft tissues is a challenging problem. We describe a method of addressing the non-union using quadriceps splitting approach to the distal femur.

Methods: We retrospectively reviewed 5 patients with distal femur infected nonunion and knee stiffness, who, after infection control, required distal femur bone grafting. All patients had autogenous iliac crest bone grafting of the distal femur using the quadriceps splitting approach. The parameters assessed were the time to surgical wound healing, wound infection, time to bony union, and if any additional procedures were needed.

Results: 5 patients were referred with distal femur infected non-union in addition to knee stiffness, with or without an implant in situ. All patients underwent debridement, implant exit, and external fixation of the femur spanning the knee as the primary surgery here, followed later on by iliac crest bone grafting of the distal femur using the above approach. All patients united well within 12 to 16 weeks, without the need for additional procedures.

Conclusions: In the presence of pre-existing knee stiffness with severely scarred and contracted soft tissues the quadriceps-splitting approach is a useful method to address bony problems in the distal femur, without the need for a separate procedure for soft tissue or flap cover.
\end{abstract}

Keywords: Infected non-union, Distal femur, Quadriceps-splitting

\section{INTRODUCTION}

Infected nonunion of the distal femur is an entity that is difficult to treat, which when coupled with severely scarred and contracted soft tissues, becomes a major orthopedic challenge. ${ }^{1}$ Staged treatment often becomes necessary and sometimes patients may need multiple visits to theatre, with or without the assistance of plastic surgery for soft tissue cover. $^{2}$ We retrospectively reviewed five patients who had presented to us with an infected distal femur non-union, who after staged treatment required bone grafting to achieve bony union. This was done using the midline quadriceps-splitting approach, which is described subsequently.
All patients had autologous iliac crest bone grafting of the distal femur using the quadriceps splitting midline approach. The objectives of the study were to assess the time to surgical wound healing, wound infection if any, time to bony union and if any additional procedures were needed.

\section{METHODS}

This is a retrospective study, done at a tertiary care centre in South India (Unit-1, Department of Orthopedics, Christian Medical College, Vellore). From January 2009 to January 2013, 5 patients who had presented to our institute with infected nonunion of the distal femur were 
included in the study. 4 of these had been referred from elsewhere, and had been operated upon in various centres, while 1 patient had been under our care from the time when he had first presented acutely to the A\&E with multiple skeletal injuries. This patient had sustained polytrauma - traumatic brain injury with fractures of the right acetabulum, right proximal femur sub trochanteric region, right distal femur open fracture, right leg midshaft open tibia-fibula fracture and left humerus closed fracture. The right distal femur open fracture eventually became an infected nonunion and required staged treatment.

All 5 patients OP and IP records, operation notes, imaging results and investigations were accessed from the medical records and patients were contacted for follow up. Preoperatively the patients had received detailed counseling regarding the proposed plan of treatment - prolonged duration of treatment, possible multiple surgical procedures, risk of recurrence of infection, no definite guarantees on the eventual range of movement of the knee, the need for close follow-up, the financial implications in the Indian scenario where the patient would need to fund his or her own treatment, including the possibility of needing higher antibiotics based on the culture reports which could also be a significant financial burden. Patients were also told about the possibility of the proposed treatment not yielding the desired results, and potentially ending up in an amputation. 2 patients had to be excluded as they did not want limb salvage, both of whom had been operated upon a total of 4 times each elsewhere and still had persistent infection with nonunion. Both of them requested for an amputation. The initial surgery in all the five patients was debridement with implant exit and knee spanning external fixation with or without local antibiotic beads. Antibiotics were given based on the intra-op culture reports and for duration of at least 6 weeks where feasible, as per the advice given by the hospital's Infectious Diseases specialists. After control of local infection based on clinical and lab-based findings, all patients underwent a $2^{\text {nd }}$ surgery - bone grafting of the distal femur. This was done through the approach described here.

\section{Operative technique}

The same team was involved in the treatment of all the patients. The corresponding author was directly involved in the treatment of 4 of the 5 patients. All the patients were already on a knee-spanning external fixator which was done as the primary surgical intervention, when they underwent bone grafting of the distal femur through this midline approach. Patients were placed supine on the operating table, with a sandbag under the ipsilateral buttock to facilitate harvesting of the bone graft from the iliac crest. After prepping and draping, a midline skin incision was made at the level of the nonunion. Thick flaps were raised. No attempt was made to delineate tissue planes. The quadriceps were split in the midline, and retracted to either side using Hohmann retractors. The non-union was adequately visualized and cleared till good bleeding bone was seen and then cortico-cancellous bone graft harvested from the ipsilateral iliac crest was grafted into the nonunion. Wound was closed in layers, with or without a suction drain. Patients were allowed toe-touch weight bearingfor the $1^{\text {st }} 6$ weeks following surgery, after which weight bearing as tolerated was allowed. X-rays were done immediate post-op and at 6 to 8 weeks follow up periods (Figure 1).

\section{RESULTS}

There were 5 patients all males, in the age-group ranging from 21 to 43 . 4 of them were healthy with no comorbidities while one patient was a diabetic, chronic smoker and a consumer of alcohol. The left femur was involved in 2 while the remaining three patients had an infected nonunion of the right distal femur. 4 were involved in road traffic accidents while 1 patient had a gunshot injury. All 5 patients had pre-existing knee stiffness and severely contracted soft tissues in the region of the distal thigh and knee prior to this procedure (Table 1).

The average number of surgical procedures prior to the initial debridement surgery in our institute was 2.4 (range 1 to 4 ). All patients had a stiff knee, and had scarred soft tissues. Bone grafting through this approach was the $2^{\text {nd }}$ surgical procedure done by us in the institute around 8 to 12 weeks following the initial debridement.

Table 1: Demographic data.

\begin{tabular}{|llllll|}
\hline Characteristics & Patient 1 & Patient 2 & Patient 3 & Patient 4 & Patient 5 \\
\hline Age & 21 & 31 & 43 & 27 & 25 \\
\hline Sex & Male & Male & Male & Male & Male \\
\hline Side & Left & Right & Right & Right & Left \\
\hline Comorbidities & Nil & CLD* & Nil & Nil & Nil \\
\hline Habits & Nil & Smoking and alcohol & Nil & Nil & Smoking \\
\hline MOI & Gunshot & RTA & RTA & RTA & RTA \\
\hline Time to presentation & 3 months & 6 months & 0 months & 4 months & 8 months \\
\hline LEFS & $60 / 80$ & $62 / 80$ & $50 / 80$ & $58 / 80$ & $45 / 80$ \\
\hline
\end{tabular}

*CLD= chronic liver disease, $\mathrm{LEFS}=$ lower extremity functional scale, $\mathrm{MOI}=$ mechanism of injury, $\mathrm{RTA}=$ road traffic accident. 

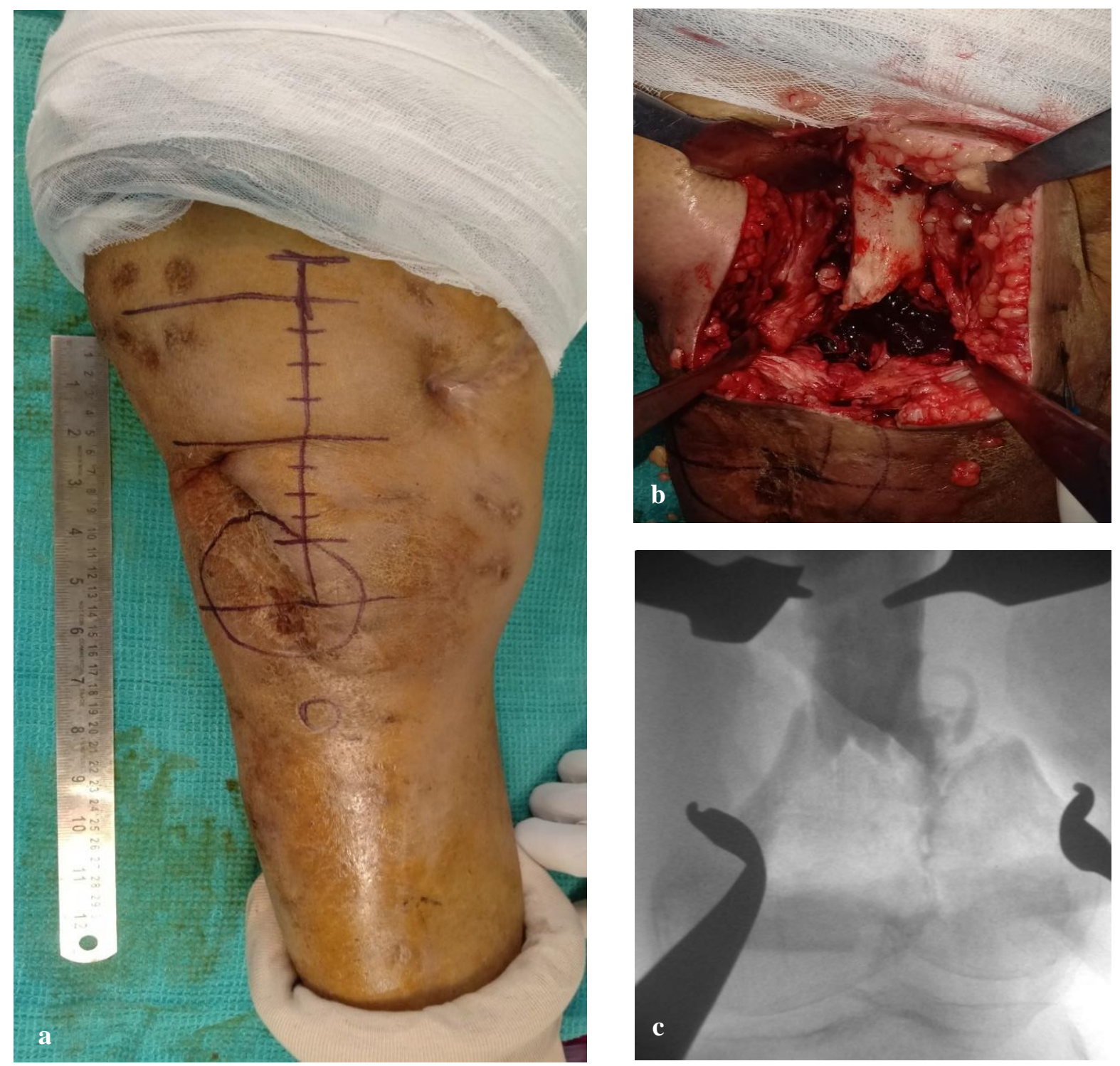

Figure 1: (a) Pre-op marking, (b) intra-op exposure with standard placement of retractors, (c) image intensification picture of the same depicting the mediolateral and superoinferior extent of visualisation possible through the above exposure.
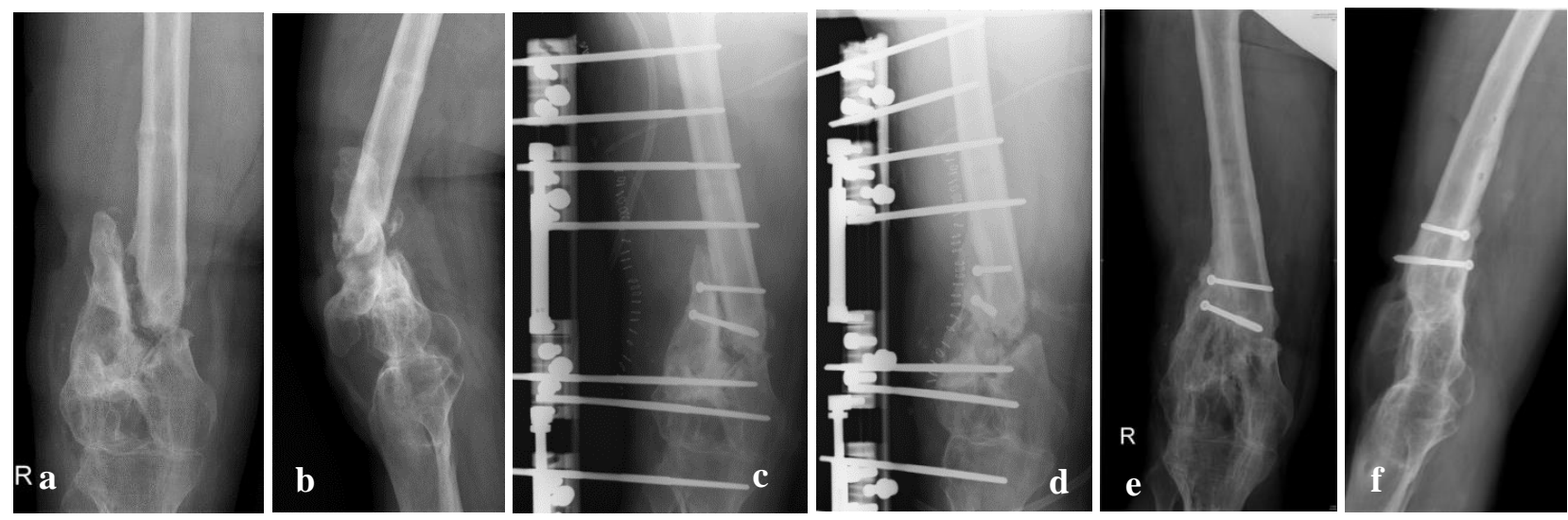

Figure 2: Radiographs: (a and b) pre-op, (c and d) immediate post-op, (e and f) final follow-up (8 years). 
All patients wound cultures showed the growth of at least 2 microorganisms. One patient had 4 bacteria isolated in his cultures. E. coli was isolated in all 5 patients, resistant to carbapenem in 2 of them.

All patients' surgical wounds healed uneventfully within an average duration of 2 weeks, there were no surgical site infection encountered with this approach. None of the patients required any further procedures to achieve union.
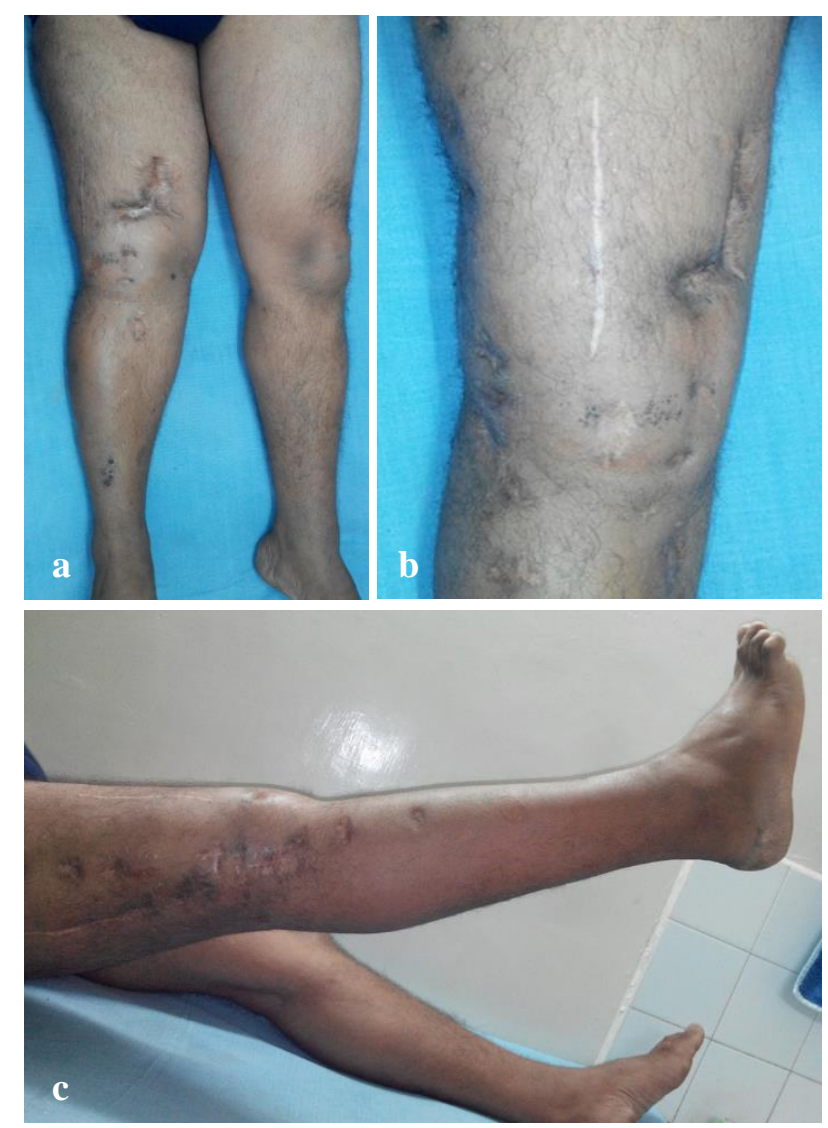

Figure 3: Final follow-up clinical pictures, (a) both lower limbs-multiple scars visualized, (b) present approach wound healed primarily, (c) patient performing straight leg raise.

All patients' fractures united without any additional surgical procedures within a period ranging from 12 to 22 weeks. None had any pin tract related problems. The external fixators were removed thereafter at an average time of 16 weeks post bone grafting. All patients continued to have knee stiffness (Figure 2 and 3) The average lower extremity functional scale at final followup was 55. The functional staging of the patients based on their lower extremity functional scale scores were that all of them were Independent community ambulators. ${ }^{3}$

\section{DISCUSSION}

In a small series of patients with infected nonunion of the distal femur in the presence of severely scarred soft tissues, we describe a novel method of addressing bony problems in the distal femur. Various approaches to the distal femur have been described extensively including the posterolateral approach, the medial approach, midline approach with either medial or lateral parapatellar window, swashbuckler technique, tibial tubercle osteotomy for better visualization, VY quadsplasty, quads snip and the Olerud extensile approach including modifications etc, most of which have been described for acute fractures and knee arthroplasties which require some amount of knee flexion during the surgery for optimal reduction and fixation. ${ }^{4-7}$

For resistant distal femur non-unions several authors have described various treatment modalities including medial plate augmentation, fibula strut grafting, allografting and megaprosthesis etc., involving various techniques. ${ }^{8-12}$ But to the best of our knowledge no technique has been described to access the distal femur in the presence of severely scarred soft tissues with a stiff knee, without having to do an osteotomy of the tibial tubercle.

All the patients in this study had an infected nonunion, treated with debridement initially followed by bone grafting through the quads-splitting approach. The end result was solid bony union in all 5 , but at the expense of knee stiffness. This had been clearly explained to all the patients. Limitations include the small number of patients, staged surgical treatment and inability to restore the knee movement.

\section{CONCLUSION}

In the presence of pre-existing knee stiffness with severely scarred and contracted soft tissues the Quadriceps - splitting approach is a useful tool to address Nonunion in the distal femur, without the need for a separate procedure for soft tissue or flap cover.

Funding: No funding sources

Conflict of interest: None declared

Ethical approval: The study was approved by the institutional ethics committee

\section{REFERENCES}

1. Struijs RWPPA. Infected Nonunion of the long bones. J Orthop Trauma. 2007;21(7):507-11.

2. Whitney TM, Heckler FR, White MJ. Gastrocnemius muscle transposition to the femur: how high can you go? Ann Plast Surg. 1995;34(4):415-9.

3. Wang YC, Hart DL, Stratford PW, Mioduski JE. Clinical Interpretation of a Lower-Extremity Functional Scale-Derived Computerized Adaptive Test. Phys Ther. 2009;89(9):957-68.

4. Garvin KL, Scuderi G, Insall JN. Evolution of the Quadriceps Snip. Clin Orthop Relat Res. 1995;321:131-7.

5. Gwathmey FW, Jones-Quaidoo SM, Kahler D, Hurwitz S, Cui Q. Distal femoral fractures: current 
concepts. J Am Acad Orthop Surg. 2010;18(10):597-607.

6. Whiteside LA. Exposure in Difficult Total Knee Arthroplasty Using Tibial Tubercle Osteotomy. Clin Orthop Relat Res. 1995;(321):32-5.

7. Khalil AE-S, Ayoub MA. Highly unstable complex C3-type distal femur fracture: can double plating via a modified Olerud extensile approach be a standby solution? J Orthop Traumatol. 2012;13(4):179-88.

8. Chapman MW, Finkemeier CG. Treatment of Supracondylar Nonunions of the Femur with Plate Fixation and Bone Graft. JBJS. 1999;81(9):1217-8.

9. Kanakeshwar RB, Jayaramaraju D, Agraharam D, Rajasekaran S. Management of resistant distal femur non-unions with allograft strut and autografts combined with osteosynthesis in a series of 22 patients. Injury. 2017;48:14-7.

10. Vaishya R, Singh AP, Hasija R, Singh AP. Treatment of resistant nonunion of supracondylar fractures femur by megaprosthesis. Knee Surg Sports Traumatol Arthrosc Off J ESSKA. 2011;19(7):1137-40.

11. Ebraheim NA, Buchanan GS, Liu X, Cooper ME, Peters N, Hessey JA, et al. Treatment of Distal Femur Nonunion Following Initial Fixation with a Lateral Locking Plate. Orthop Surg. 2016;8(3):32330.

12. Wang J-W, Weng L-H. Treatment of distal femoral nonunion with internal fixation, cortical allograft struts, and autogenous bone-grafting. J Bone Jt Surg Am Vol. 2003;85(3):436-40.

Cite this article as: Bliss J, Inja DB, Nithyananth M, Cherian VM. Quadriceps-splitting midline approach in the treatment of distal femur infected nonunion with stiff knee and severely scarred soft tissues. Int J Res Orthop 2020;6:276-80. 